\title{
Spontaneous perforated pyometra presenting as an acute abdomen: appraisal of three consecutive cases
}

\author{
Pooja Paudyal, Josie Baral, Geeta Gurung, Ashma Rana, Suniti Rawal, Meeta Singh \\ Dept. of Obstetrics and Gynaecology, Tribhuvan University Teaching Hospital, Kathmandu, Nepal.
}

Correspondence: Pooja Paudyal

Email: paudyalpooja@yahoo.com

\begin{abstract}
Introduction: Pyometra is the accumulation of purulent fluid in the uterine cavity caused by impaired drainage of pus due to benign or malignant conditions; rarely with resultant spontaneous uterine perforation brought about by uterine distension.

Cases: We report three consecutive cases of spontaneous perforated pyometra in postmenopausal elderly women aged 60 to 70 years who presented with acute abdomen, initially diagnosed as hollow viscus perforation, demonstrated of having significant uterine perforation at laparotomy for which hysterectomy with bilateral salpingo-oophorectomy were done. Histopathological examination revealed features of necrosis of stroma with chronic endometritis without any evidence of malignancy in all the three cases.

Conclusion: Spontaneous perforated pyometra, a grave complication necessitating prompt intervention, should always be kept as a possibility in all cases of suspected pyoperitoneum and acute abdomen in elderly postmenopausal women.
\end{abstract}

Keywords: Acute abdomen; Perforated pyometra; Spontaneous.

\section{Introduction}

Pyometra, an uncommon gynecologic entity, is the accumulation of purulent fluid in the uterine cavity caused by impaired drainage of pus due to benign or malignant

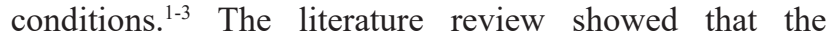
incidence of pyometra was $0.2 \%$ to $0.5 \%$ in all gynecologic admissions and occurred in $13.6 \%$ disorders of elderly gynecologic outpatients. ${ }^{4}$ Spontaneous perforation of uterus is a very rare complication of pyometra, but it must be considered in the differential diagnosis of peritonitis in elderly patients. Early diagnosis is very important in starting immediate treatment, thus reducing morbidity and mortality.

\section{Cases}

\section{Case 1}

60 years Para 7 postmenopausal lady presented with sudden onset pain abdomen for 2 days, had generalized tenderness, guarding and rigidity on examination. Ultrasonogram
(USG) showed acute appendicitis with collection in peritoneal cavity likely appendicular perforation. She underwent emergency exploratory laparotomy where $200 \mathrm{ml}$ of pus was drained from peritoneal cavity, appendix and rest of abdominal viscera were normal but a $3 \times 2 \mathrm{~cm}$ perforation over anterior wall of uterus was noted just below the fundus with unhealthy margin. Subtotal hysterectomy with bilateral salpingo-oophorectomy (TAH with BSO) was performed. Pus culture revealed E. coli. Patient developed burst abdomen on $12^{\text {th }}$ post-operative day. Histopathologic examination (HPE) showed chronic endometritis with chronic cervicitis.

\section{Case 2}

64 years post-menopausal Para 4+1 lady presented with pain abdomen for one week which was more severe since one day with episodes of nausea and vomiting. She also had on and off history of per vaginal discharge since one year. On examination she had features of acute abdomen 
and USG was suggestive of hollow viscus perforation. On emergency laparotomy there was pyoperitoneum with about $4 X 3 \mathrm{~cm}$ eaten up area in the fundic region of uterus covered with slough. She was managed with TAH with BSO. The HPE showed presence of necrotic tissue in the stroma with absence of features of malignancy.

\section{Case 3}

70 years Para 8 postmenopausal lady presented with fever and pain abdomen for 10 days with gross distension and tenderness of the abdomen on examination. Her investigations revealed raised Total Count (TC) with neutrophilia and USG showed minimal free fluid in lesser sac, perihepatic region and on aspiration yielded purulent fluid. Emergency exploratory laparotomy was performed with findings of $1.5 \mathrm{~L}$ pus present in peritoneal cavity, no collection in lesser sac, no hollow viscus perforation. A $2 \times 2$ $\mathrm{cm}$ perforation was noted in fundus of uterus with black and necrotic edges of rent. TAH with BSO was done with peritoneal lavage. HPE report showed acute endometritis with ulceration and chronic cervicitis.

All three patients had ICU admission, prolonged hospital stay, needed higher antibiotics and one patient developed burst abdomen and hence needed relaparotomy. Though we prevented mortality in all three women, there was significant morbidity.

\section{Discussion}

Pyometra usually develops in elderly women. It is thought to be caused by occlusion of the cervical canal secondary to chronic cervicitis, radiation cervicitis, benign or malignant cervical lesions, puerperal infections, foreign bodies, or congenital anomalies. ${ }^{2,3}$ Also, the risk factors may include a decline in activity, incontinence, diabetes, and long-term intrauterine device use. ${ }^{5}$ The classic triad of symptoms in patients with pyometra consists of purulent vaginal discharge, postmenopausal bleeding, and lower abdominal pain. However, more than $50 \%$ of all patients with nonruptured pyometra are asymptomatic. ${ }^{6}$ Though spontaneous perforation of uterus is rare it can be catastrophic resulting in significant morbidity and mortality. Once ruptured, the symptoms become severe and acute abdomen often develops; it is therefore important to make a differential diagnosis from other causes of acute surgical abdomens in elderly patients. In all three cases initial diagnosis was that of hollow viscus perforation and diagnosis of ruptured pyometra was made only on laparotomy. Their management is often difficult and hysterectomy and bilateral salpingo-oopherectomy may be the best choice procedure in these patients. ${ }^{7}$

\section{Conclusion}

Spontaneous perforated pyometra, a grave complication necessitating prompt intervention, should always be kept as a possibility in all cases of suspected pyoperitoneum and acute abdomen in elderly postmenopausal women.

\section{References}

1. Ou YC, Lan KC, Lin H, Tsai CC, ChangChien CC. Clinical characteristics of perforated pyometra and impending perforation: specific issues in gynecological emergency. J Obstet Gynaecol Res. 2010;36(3):661-6.

2. Vyas S, Kumar A, Prakash M, Kapoor R, Kumar P, Khandelwal N. Spontaneous perforation of pyometra in a cervical cancer patient: a case report and literature review. Cancer Imaging. 2009;9:12-4.

3. Lee SL, Huang LW, Seow KM, Hwang JL. Spontaneous perforation of a pyometra in a postmenopausal woman with untreated cervical cancer and "forgotten" intrauterine device. Taiwan J Obstet Gynecol. 2007;46(4):439-41.

4. Ikeda M, Takahashi T, Kurachi H. Spontaneous perforation of pyometra: a report of seven cases and review of the literature. Gynecol Obstet Invest. 2013;75(4):243-9.

5. Inui A, Nitta A, Yamamoto A, Kang SM, Kanehara I, Tanaka H,et al. Generalized peritonitis with pneumoperitoneum caused by the spontaneous perforation of pyometra without malignancy:report of a case. Surg Today 1999;29:935-8.

6. Nuamah NM, Hamaloglu E, Konan A. Spontaneous uterine perforation due to pyometra presenting as acute abdomen. Int J Gynaecol Obstet 2006;92:145-6.

7. Nakao A, Mimura H, Fujisawa K, et al. Generalized peritonitis due to spontaneously perforated pyometra presenting as pneumoperitoneum: report of a case. Surg Today. 2000;30(5):454-457. 